\title{
Interpretation of the Protocol for Prevention and Control of COVID-19 in China (Edition 8)
}

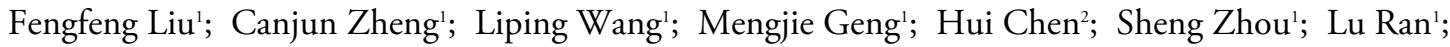 \\ Zhongjie Li'; Yanping Zhang'; Zijian Feng'; George F. Gao'; Zhaorui Chang ${ }^{1, *}$
}

\section{BACKGROUND}

Since the release of Protocol for Prevention and Control of COVID-19 (Edition 7), coronavirus disease 2019 (COVID-19) control and prevention has been facing new challenges in China with evolving domestic and global epidemiological situations. Since September 2020, China has experienced more than 20 local outbreak waves, all of which were able to be contained within a few weeks. Investigation and response to these importation-related outbreaks not only resulted in the accumulation of rich experience, but also exposed new problems requiring remediation. First, rural areas are potential weak links in epidemic prevention and control. Second, prevention of coronavirus importation and transmission risk is not limited to incoming travelers from overseas, as imported coldchain food and goods and people with occupational exposure to these goods are now known to be able to cause local outbreaks. Third, there are weaknesses in the management of centralized quarantine. In addition to these three identified risks, another major change since publication of the seventh edition is that several COVID-19 vaccines developed by China have been granted conditional market authorization or emergency use approval by the National Medical Products Administration. In December 2020, China officially launched a large-scale vaccination campaign using the approved vaccines (1). Vaccination adds strength and new characteristics to the prevention and control of COVID-19.

The National Health Commission (NHC) and external experts worked together to update the seventh edition of the prevention and control protocol to an eighth edition based on carefully evaluated experience with the previous containment measures, and with anticipation of matters related to COVID-19 vaccination. In this article, we provide our interpretation of key revisions and updates to the seventh edition that can be found in the eighth edition of the protocol.

\section{STRUCTURE}

The new edition strengthens technical guidance for grassroots-level work. The protocol now has nine sections that describe work requirements, including general requirements, the etiological and epidemiological characteristics, public measures, epidemic surveillance methods, outbreak response, laboratory-based detection, prevention and control of imported viruses, strengthen the prevention and control of key links, and organizational responsibilities. The new protocol also includes 12 detailed technical guidelines as attachments that provide information for prevention and control personnel in a variety of occupations.

\section{IMPROVEMENTS IN EPIDEMIC CONTAINMENT}

The protocol emphasizes early prevention, detection, reporting, quarantine, and treatment; adheres to the principle of preventing simultaneous transmission from people and goods; and enhances prevention of transmission from contaminated, imported cold-chain food and goods. It strengthens epidemic prevention and control during key periods of time, including holidays, and in key areas, including rural areas, to detect sporadic cases and clusters of cases as early as possible and to achieve precise, powerful, orderly, and effective outbreak response.

\section{STRENGTHEN VACCINATION AND PREVENTION AND CONTROL AFTER VACCINATION}

Vaccination is the most effective method for preventing, controlling, and ultimately defeating COVID-19 virus, also known as severe acute 
respiratory syndrome coronavirus 2 (SARS-CoV-2). The new edition emphasizes vaccination in the sections on "public measures" and "prevention and control of key populations." The current work and strategies for COVID-19 vaccination in China are to complete vaccination of key population aged 18 years or above at high risk of occupational exposure to SARS-CoV-2; to continue vaccination of citizens traveling abroad to areas with ongoing transmission; to vaccinate domestic populations at high risk of transmission in key regions of the country, service industry workers, and workers in labor-intensive industries; and to offer vaccination to all individuals 18 years or above who would like to be vaccinated and have no contraindications to vaccination. Domestic vaccination strategies and their management will be adjusted and improved in a timely manner based on progress of vaccine research and results of clinical trials and observational studies.

Because COVID-19 vaccination results in an antibody response in the person vaccinated, the current diagnostic and exclusion criteria for cases have been adjusted so that serum $\operatorname{IgM}$ and IgG antibody tests are no longer used as indicators for diagnosis or exclusion. To continuously monitor COVID-19 vaccine protection effectiveness and safety, the eighth edition of the protocol includes gathering vaccine administration data on anyone identified as infected, regardless of the presence of symptoms. Additionally, sequencing viruses isolated from infected individuals will enable the study of SARS-CoV-2 variants and the ability of COVID-19 vaccines to provide protection from variants.

\section{STRENGTHEN EPIDEMIC SURVEILLANCE AND PROMPTLY IDENTIFY RISK FACTOR}

Sensitive monitoring and early warning systems are essential for early detection and effective outbreak response. To further improve detection, identification, and early warning of cases, the protocol continues to strengthen case detection sensitivity of medical institutions, multi-channel surveillance, and early warning mechanisms. The previous outbreaks indicated that index cases in the outbreaks were best detected by medical institutions. Examples of medical institutions identifying outbreak index cases include the Xinfadi Wholesales Market outbreak in Beijing and the Dalian, Shanghai Pudong Airport, Qingdao, and
Manchuria outbreaks that occurred in 2020 (2). In contrast, it was found that community health service stations, village clinics, and private clinics did not have nucleic acid testing capabilities and were weak links in case detection in the outbreaks that occurred in Shijiazhuang and Shenyang. To address this weakness, the eighth protocol expands the scope of nucleic acid testing for patients attending any medical institution - nucleic acid testing is required for all patients with fever. For individuals without fever who have COVID-19-related symptoms such as dry cough, weakness, sore throat, hyposmia/hypogeusia, or diarrhea, nucleic acid testing is to be performed for those with an epidemiological history compatible with COVID-19 and those working in high-risk occupations (e.g., medical staff receiving patients with fever or infectious diseases, individuals engaged in cold-chain food supervision and related work, and management and service personnel at quarantine sites).

When patients with fever or other COVID-19 related symptoms are identified in community health services stations, village clinics, or private clinics, patient information is required to be reported to the community healthcare center or township health center within 2 hours. A nucleic acid testing strategy of "reporting by village level medical institutions, sampling by township level medical institutions, and detecting by county level medical institutions" is to be implemented to ensure the earliest possible case detection.

To promptly identify risks and early warning signs and guide local government implementation of multichannel monitoring, the protocol refines requirements and methods for monitoring of people, goods, and the environment. The scope includes patients attending medical institutions, individuals engaged in occupations with infection risk, health monitoring of key populations, monitoring of key institutions, monitoring of imported goods, and environmental sample monitoring in centralized quarantine sites, medical institutions, and wet markets and sewage from large processing facilities for imported frozen goods.

To facilitate understanding of the prevalence of SARS-CoV-2 variants and their influence on laboratory detectability and vaccine effectiveness, the new protocol strengthens pathogen monitoring and includes monitoring variants of concern and variants of interest, as designated by the World Health Organization (3). 


\section{STANDARDIZE OUTBREAK MANAGEMENT AND RESPONSE}

The protocol summarizes outbreak response experiences and standardizes relevant information in ten aspects: infection source control, epidemiological investigation and tracing to the infectious source, close contact tracing and management, nucleic acid testing of key populations, patients and close contact transportation, centralized quarantine site management, community (village) management and control, disinfection, mental health services, and release of epidemic information. In response to rural area issues, such as poor medical conditions, inadequate capacity for nucleic acid testing, and limited quarantine sites, the updated protocol provides guidance for containment measures targeted to rural areas.

\section{STRICT MANAGEMENT OF QUARANTINE FOR MEDICAL OBSERVATION}

A few incoming international travelers test nucleicacid-positive after 14 days of centralized quarantine. To prevent this residual transmission risk, the eighth edition protocol enhances management of quarantine for travelers entering China and the close contacts of COVID-19 virus infected cases based on a comprehensive analysis of possible causes. Incoming travelers from overseas and all the close contacts of COVID-19 virus infected cases are still required to be quarantined for 14 days in designated centralized quarantine sites. Nucleic acid tests are performed using nasopharyngeal swab samples on days 1, 4, 7, and 14 of quarantine. Two nasopharyngeal swabs are to be collected at the time of discharge or release from quarantine and tested using different reagents and by different institutions in principle. After release from quarantine, travelers and close contacts are required to stay at home for seven days of health monitoring, with the nucleic acid tests performed on days 2 and 7 . During the household health monitoring period, individuals should adhere to health monitoring, personal protection, staying at home, and not participating in any activities involving gatherings of people.

\section{PRECISE MANAGEMENT OF CLOSE CONTACTS OF CLOSE CONTACTS}

Due to the relatively low risk of infection and transmission from close contacts of close contacts (secondary close contacts) and to ensure the effectiveness of epidemic prevention and control, the protocol adjusts management of close contacts of close contacts to reduce pressure on quarantine sites and management tasks for clusters of infection. The duration of quarantine is determined from the actual situations of close contacts of close contacts. If a close contact is released from quarantine, his/her close contacts may also be released from quarantine; if a close contact has negative results from the first two nucleic acid tests, his/her close contracts may be released from quarantine if they have negative nucleic acid tests on days 1,4 , and 7 .

\section{SIMULTANEOUS PREVENTION OF INFECTION FROM PEOPLE AND GOODS}

The global pandemic situation remains severe, as epidemics have rebounded in some countries. Additionally, virus variants have increased pressure on preventing importation of the virus in China. The tracing to the infectious source of previous outbreaks indicated that imported cases and cold-chain foods and goods can cause local spread and epidemics. In addition to the management of oversea travelers entering China, the new protocol requires management of imported goods, management of persons in direct contact with imported goods, and control measures for positive testing goods and people in contact with these goods.

Specific measures are as follows: 1) strengthen sampling and testing of imported cold-chain foods, their processing environments, transportation, storage, and sales, and strictly implement prevention and control and tracing management during the entire process; 2) strengthen sampling, testing, and preventive disinfection of imported, high-risk, non-cold-chain goods at ports; 3) in addition to regular health education and health monitoring, nucleic acid testing is required to be conducted once a week and before leaving a job for people in direct contact with imported goods at ports; 4) when imported good are identified as positive for COVID-19 virus, temporary storage and safe disposal should be practiced, and the working area 
is required to be disinfected. According to a transmission risk assessment, health monitoring and nucleic acid testing will be conducted for personnel contacting goods that test positive. Centralized medical quarantine can be used for contacts when necessary.

\section{DISCUSSION}

Building on the previous seven editions of the protocol for prevention and control of COVID-19, the eighth edition adheres to an overall containment strategy of "preventing imported virus and domestic resurgence," and summarizes relevant technical documents issued previously and the experience in managing local outbreaks. The protocol covers all aspects of containment measures and integrates administrative management with technical implementation. It is a practical reference book to guide all localities in their response to COVID-19.

The seventh edition of the protocol played an important role in the prevention and control of the epidemic during autumn and winter of 2020. However, factors such as vaccine availability, discoveries of SARS-CoV-2 variants, and resurgences in other countries have brought new challenges for continued epidemic containment in China. The eighth edition improves and revises key containment measures, including vaccination, epidemic surveillance, outbreak response, quarantine management, management of close contacts of close contacts, and prevention and control of imported virus. The protocol will continue to be updated to provide technical support for achieving China's goals for containment of COVID-19 based on epidemic situations at home and abroad, vaccination in China, and scientific advances on variants of SARS-CoV-2, development of drugs, and other topics relevant to ending the pandemic and returning to a more normal global societal situation.

doi: $10.46234 / \mathrm{ccdcw} 2021.138$

\# Corresponding author: Zhaorui Chang, changzr@chinacdc.cn.

${ }^{1}$ Division of Infectious Disease, Key Laboratory of Surveillance and Early Warning on Infectious Disease, China CDC, Beijing, China; ${ }^{2}$ National Center for Tuberculosis Control and Prevention, China CDC, Beijing, China; ${ }^{3}$ Chinese Center for Disease Control and Prevention, Beijing, China.

Submitted: June 09, 2021; Accepted: June 12, 2021

\section{REFERENCES}

1. National Health Commission of China. Transcript of the press conference of the Joint Prevention and Control Mechanism of the State Council on December 19, 2020. http://www.nhc.gov.cn/xcs/s3574/ 202012/dd603e667f6d47238a8998860f8b9c0a.shtml. [2021-6-5]. (In Chinese).

2. Li ZJ, Liu FF, Cui JZ, Peng ZB, Chang ZR, Lai SJ, et al. Comprehensive large-scale nucleic acid-testing strategies support China's sustained containment of COVID-19. Nat Med 2021;27(5):740 - 2. http://dx. doi.org/10.1038/s41591-021-01308-7.

3. WHO. SARS-CoV-2 variants of concern and variants of interest, updated 31 May 2021. https://www.who.int/en/activities/trackingSARS-CoV-2-variants/. [2021-6-5]. 